\title{
Some Dido-type Inequalities
}

\begin{abstract}
Alan Siegel
Alan Siegel is the product of the Palo Alto public school system in California. He earned his B.S. in mathematics and his Ph.D. in computer science at Stanford University. He is presently the Deputy Chairman for the Department of Computer Science at New York University. His areas of specialty include the mathematical analysis of algorithms, probabilistic analysis, and lower bounds. He is also a cofounder of a project that seeks to improve mathematics education in the U.S.
\end{abstract}

\section{Introduction}

The standard Isoperimetric Theorem for polygons with a fixed set of edge lengths is as follows (cf. [2]):

Theorem 0 Let $S$ be a finite multiset of positive values. Then among all polygons with edge lengths that comprise the elements of $S$, those that are inscribed in a circle have the greatest area.

Of course, Theorem 0 includes the implicit restriction that the largest value in $S$ does not exceed the sum of the other values, since no polygon could have such a longest edge.

Now suppose that we are given a collection of line segments with fixed lengths, and wish to arrange them to form an open convex path whose convex hull has the greatest possible area. The solution to this optimization problem is also well-known. The answer is to place the segments consecutively so that they are inscribed in a semicircle of suitable minimum radius. The resulting path, and the polygon that is formed by including the diameter of the enclosing semicircle solve this area maximization problem. This fact follows

Bekanntlich schliesst der Kreis bei vorgelegtem Umfang die grösste Fläche ein. Der Beweis dieser und analoger Problemstellungen wird mit Hilfe isoperimetrischer Ungleichungen geführt. Grundkenntnisse zu dieser Thematik waren offenbar bereits im Altertum vorhanden. So wird in Vergils Aeneis über die Gründerin Karthagos, Königin Dido, berichtet (erster Gesang, Verse 367/368): "Dort erstanden sie Land, von dem Handel einst Byrsa geheissen, was mit der Haut des Stiers sie einzuschliessen vermochten". Im vorliegenden Beitrag führt uns der Autor mit Hilfe elementarer Methoden zur Lösung eines isoperimetrischen Problems, bei dem nicht nur die Länge, sondern auch die geometrische Form des Umfangs vorgegeben ist. $j k$ 
from the Reflection Principle and Theorem 0. Accordingly, we define the following area maximization function.

Definition 1 Let $S$ be a finite multiset of positive numbers. Let $D$ Area $(S)$ equal the area of such a maximally sized polygon formed from edges with the lengths in $S$, plus one extra free edge as described above.

In 1989, A. and K. Bezdek formulated a new but related area maximization problem as the following Dido-type theorem [1].

Theorem 1 Let $\widetilde{S}$ be a physical arrangement of segments in $\mathbb{R}^{2}$ that is pointwise connected, and let the multiset $S$ comprise the lengths of the segments in $\widetilde{S}$. Then

$$
\operatorname{Area}(\operatorname{ConvHull}(\widetilde{S})) \leq \operatorname{DArea}(S) \text {. }
$$

To be precise, Bezdek and Bezdek formulated this statement as a conjecture, and proved the result if $\widetilde{S}$, when considered as a graph with vertices and edges, is connected [1]. Now, their formulation does allow edges to cross each other, but requires that the graph connectivity, which is a consequence of edges sharing vertices as endpoints, must comprise a single component. They conjectured that this restriction should be unnecessary.

\section{The inequalities}

We show that their conjecture is indeed correct. We also give two generalizations of their theorem that, we believe, enable this result to be proven quite easily.

Definition 2 Let $\widetilde{S}=\left(\widetilde{s}_{1}, \widetilde{s}_{2}, \ldots, \widetilde{s}_{n}\right)$ be a sequence of $n$ physically placed segments in $\mathbb{R}^{2}$. We say that $\widetilde{S}$ is weakly connected if, for $j=1,2, \ldots, n-1$ :

$$
\tilde{s}_{j+1} \cap \operatorname{ConvHull}\left(\tilde{s}_{1}, \tilde{s}_{2}, \ldots, \tilde{s}_{j}\right) \neq \emptyset .
$$

This definition immediately suggests the following theorem.

Theorem 2 Let $\widetilde{S}$ be a weakly connected sequence of segments in $\mathbb{R}^{2}$, and let the multiset $S$ comprise the lengths of the segments in $\widetilde{S}$. Then

$$
\operatorname{Area}(\operatorname{ConvHull}(\widetilde{S})) \leq \operatorname{DArea}(S) \text {. }
$$

Obviously, Theorem 2 implies Theorem 1. With one intermediate step, the chain of reasonings becomes a matter of a few elementary observations.

Definition 3 We say that a sequence of physically placed segments $\left(\tilde{s}_{1}, \tilde{s}_{2}, \ldots, \tilde{s}_{n}\right)$ is optimally sequenced if the following hold.

1) The sequence defines a connected path with the same natural ordering of edges as given by the sequence.

2) The path, when augmented by an edge that connects its first and last vertices, defines the boundary of a convex region.

3) The rotation of edges along the path is less than $\pi$. Formally, $\pi$ exceeds the sum of the angles that are supplementary to the interior angles of the path. 
This definition will be used in Theorem 3 to show that the best way to translate edges is to arrange them so that they are sorted by slope and connected to form a path.

Theorem 3 Let $\widetilde{S}=\left(\tilde{s}_{1}, \tilde{s}_{2}, \ldots, \tilde{s}_{n}\right)$ be a weakly connected sequence of segments in $\mathbb{R}^{2}$. Let $\widetilde{T}=\left(\tilde{t}_{1}, \tilde{t}_{2}, \ldots, \tilde{t}_{n}\right)$ be optimally sequenced, and suppose that these edges comprise a reordering and translation of the segments in $\widetilde{S}$ as necessary, with rotations precluded. Then

$$
\operatorname{Area}(\operatorname{ConvHull}(\widetilde{S})) \leq \operatorname{Area}(\operatorname{ConvHull}(\widetilde{T})) .
$$

Now, the standard Isoperimetric Theorem for a fixed set of segment lengths and a free line, as noted in the remarks following Theorem 0, shows that Area $(\mathrm{ConvHull}(\widetilde{T})) \leq$ $\operatorname{DArea}(T)$, where $T$ is the multiset comprising the lengths of the segments in $\widetilde{T}$. Since this fact gives Theorem 2, we need only prove Theorem 3 .

Proof of Theorem 3. Let the vertices of the path defined by $\widetilde{T}$ be $p_{0}, p_{1}, \ldots, p_{n}$, so that $\tilde{t}_{i}=\bar{p}_{i-1} p_{i}$. Let $\vec{v}_{i}$ be the vector that points from $p_{i-1}$ to $p_{i}$. For convenience, let the rotational sense of $\widetilde{T}$ be positive, so that the cross product formulation we use will give positive values. Let $\overrightarrow{s_{i}}$ be a vector rooted at the origin that is parallel and congruent to the segment $\tilde{s}_{i}$, for $i=1, \ldots, n$.

Let $R_{j}=\operatorname{ConvHull}\left(\tilde{s}_{1}, \tilde{s}_{2}, \ldots, \tilde{s}_{j-1}\right)$. Suppose, for the moment, that $\tilde{s}_{j}$ has just an endpoint that lies within $R_{j}$. Then adjoining $\tilde{s}_{j}$ to $R_{j}$ and taking the convex hull effectively adjoins a triangle to $R_{j}$ where the base lies within $R_{j}$ and the opposing vertex is the endpoint of $\tilde{s}_{j}$ that is exterior to $R_{j}$. The increase in area is bounded by the area of the triangle, which, in turn, is half the cross product of $\vec{s}_{j}$ and a vector that corresponds to the base.

Let $\vec{d}_{j}$ be a located vector that lies entirely within the region $R_{j}$ and that, among all such vectors, yields a maximum value for $\vec{s}_{j} \times \vec{d}_{j}$. Our reasoning shows that $\operatorname{Area}\left(R_{j+1}\right)-$ $\operatorname{Area}\left(R_{j}\right) \leq \frac{1}{2} \vec{s}_{j} \times \vec{d}_{j}$, subject to our supposition about $\tilde{s}_{j} \cap R_{j}$. To see that this inequality must hold whenever $R_{j} \cap \tilde{s}_{j} \neq \emptyset$ and $R_{j}$ is convex, observe that if $\tilde{s_{j}}$ has two subsegments that exit $R_{j}$, we can apply the above reasoning to each subsegment in turn. Since the two pieces are parallel, the same $\overrightarrow{d_{j}}$ can be used in the area estimate for each augmentation.

Summing these area differences gives

$$
\operatorname{Area}(\operatorname{ConvHull}(\widetilde{S})) \leq \frac{1}{2} \sum_{j} \vec{s}_{j} \times \vec{d}_{j}
$$

Evidently, we will get a term that is at least as large as $\vec{s}_{j} \times \vec{d}_{j}$ if we replace $\overrightarrow{d_{j}}$ in (1) by the sum $\sum_{i=1}^{j-1} u_{i, j} \vec{s}_{i}$, where $u_{i, j}=\operatorname{sign}\left(\overrightarrow{s_{j}} \times \overrightarrow{s_{i}}\right)$.

Substituting gives

$$
\begin{aligned}
\operatorname{Area}(\operatorname{ConvHull}(\widetilde{S})) & \leq \frac{1}{2} \sum_{j=1}^{n} \vec{s}_{j} \times \sum_{i=1}^{j-1} u_{i, j} \vec{s}_{i} \\
& \leq-\frac{1}{2} \sum_{i=1}^{n} \overrightarrow{s_{i}} \times \sum_{j=i+1}^{n} u_{i, j} \vec{s}_{j}
\end{aligned}
$$


Averaging these two expressions shows that

$$
\operatorname{Area}(\operatorname{ConvHull}(\widetilde{S})) \leq \frac{1}{4} \sum_{j} \vec{s}_{j} \times \vec{e}_{j}
$$

where $\vec{e}_{j}=\sum_{i=1, i \neq j}^{n} w_{i, j} \vec{s}_{i}$, and $w_{i, j}=\operatorname{sign}\left(\vec{s}_{j} \times \vec{s}_{i}\right)$.

But as the following shows, this formula is satisfied with equality by the $\widetilde{T}$ vectors:

$$
\begin{aligned}
\operatorname{Area}(\operatorname{ConvHull}(\widetilde{T})) & =\sum_{i=1}^{n} \operatorname{Area}\left(\triangle p_{i-1} p_{i} p_{n}\right) \\
& =\frac{1}{2} \sum_{i=1}^{n}\left(\vec{v}_{i} \times \sum_{j=i+1}^{n} \vec{v}_{j}\right) \\
& =\sum_{i=1}^{n} \operatorname{Area}\left(\triangle p_{i-1} p_{i} p_{0}\right) \\
& =-\frac{1}{2} \sum_{i=1}^{n}\left(\vec{v}_{i} \times \sum_{j=1}^{i-1} \vec{v}_{j}\right), \\
& =\frac{1}{4} \sum_{i=1}^{n} \vec{v}_{i} \times\left(-\sum_{j=1}^{i-1} \vec{v}_{j}+\sum_{j=i+1}^{n} \vec{v}_{j}\right)
\end{aligned}
$$

However, since the vectors are sorted by incline, and cannot have directions that differ by as much as $\pi$, the projection of $-\vec{v}_{j}$, for $j<i$ and $\vec{v}_{j}$, for $j>i$ onto a unit perpendicular to $\vec{v}_{i}$ all point in the same direction. Consequently, if $\vec{s}_{\ell}$ corresponds to $\vec{v}_{i}$, then $\vec{e}_{\ell}=\left(-\sum_{j=1}^{i-1} \vec{v}_{j}+\sum_{j=i+1}^{n} \vec{v}_{j}\right)$ provided $\vec{s}_{\ell}$ is parallel to $\vec{v}_{i}$. If the vectors are antiparallel, then $\vec{e}_{\ell}=\left(\sum_{j=1}^{i-1} \vec{v}_{j}-\sum_{j=i+1}^{n} \vec{v}_{j}\right)$. In view of inequality (2), Theorem 3 now follows.

\title{
References
}

[1] Bezdek, A., Bezdek, K.: On a discrete Dido-type question. Elem. Math. 4 (1989), 92-100.

[2] Kazarinoff, N.D.: Geometric Inequalities. New York (1961), Random House.

\author{
Alan Siegel \\ New York University \\ Department of Computer Science \\ The Courant Institute \\ 251 Mercer Street \\ New York City, NY 10012-1185, U.S.A. \\ e-mail: siegel@cs.nyu.edu
}

